\title{
Relación entre perfeccionismo y salud mental en futbolistas jóvenes: diferencias entre categorías deportivas
}

\author{
Relationship Between Perfectionism and Mental Health in \\ Young Soccer Players: Differences Between Sports Categories
}

Pedro Pérez-Hernández'

Belén Olmedilla-Caballero' 수

Verónica Gómez-Espejo'

Aurelio Olmedilla ${ }^{1,2 \star}$ (1)

1. Facultad de Psicología, Campus Regional de Excelencia Internacional "Campus Mare Nostrum", Universidad de Murcia, España. 2. Sports Performance Analysis Association. España.

\section{Resumen}

El objetivo principal del presente estudio es estudiar la relación entre el perfeccionismo y la salud mental (depresión, estrés y ansiedad) en jóvenes futbolistas, para determinar la existencia de diferencias en esta relación entre tres categorías deportivas (infantil, cadete y juvenil). Concretamente se pretende: a) determinar las diferencias en los niveles de perfeccionismo (adaptativo y desadaptativo) según la categoría deportiva: Infantil (12-13 años) Cadete (14-15 años) y Juvenil (16-18 años); b) determinar la relación entre el perfeccionismo (adaptativo vs. desadaptativo) y los indicadores de salud mental (Depresión, Ansiedad y Estrés) tanto en categoría Infantil, Cadete y Juvenil, como en el total de la muestra. Los participantes fueron 131 jugadores jóvenes de fútbol con una media de edad de 14,31 años ( $\pm 1,42) ; 50$ de ellos jugaban en categoría infantil, 44 en categoría cadete y 37 en categoría juvenil. Para evaluar el perfeccionismo se utilizó la Escala Multidimensional del Perfeccionismo (MPS), y para evaluar los indicadores de salud mental se utilizó la Escala de Depresión, Ansiedad y Estrés (DASS-21). Se realizó un análisis descriptivo de las variables y un análisis de correlación de Pearson para determinar la relación entre aquellas. Los resultados indican que los niveles de perfeccionismo total, así como los de perfeccionismo adaptativo y perfeccionismo desadaptativo, son similares en las tres categorías deportivas estudiadas. El perfeccionismo desadaptativo se relaciona positivamente con los indicadores de salud mental (Depresión, Ansiedad, y Estrés), es decir, a mayor puntuación en perfeccionismo desadaptativo mayores niveles de depresión, de estrés y de ansiedad manifiestan los jugadores. Se proponen sugerencias prácticas derivadas del estudio.

Palabras clave: Perfeccionismo, salud mental, fútbol, jóvenes, categorías deportivas

\begin{abstract}
The main objective of this study is to study the relationship between perfectionism and mental health (depression, stress and anxiety) in young football players, to determine the existence of differences in this relationship between three sports categories (under 12-13, under 14-15 and under 16-18). Specifically, the aim is to: a) determine the differences in the levels of perfectionism (adaptive and maladaptive) according to the sports category: under 12-13, under 14-15 and under 16-18; b) determine the relationship between perfectionism (adaptive vs. maladaptive) and mental health indicators (depression, anxiety and stress) both in the under 12-13, under 14-15 and under 16-18 categories and in the total sample. The participants were 131 young football players with an average age of 14.31 years ( \pm 1.42$) ; 50$ of them played in the under 12-13, 44 in the under 14-15 and 37 in under 16-18. The Multidimensional Perfectionism Scale (MPS) was used to assess perfectionism, and the Depression, Anxiety and Stress Scale (DASS-21) was used to assess mental health indicators. A descriptive analysis of the variables and a Pearson's correlation analysis were performed to determine the relationship between them. The results indicate that the levels of total perfectionism, as well as those of adaptive perfectionism and maladaptive perfectionism, are similar in the three sports categories studied. Disadaptive perfectionism is positively related to mental health indicators (Depression, Anxiety, and Stress), that is, the higher the score in disadaptive perfectionism the higher the levels of depression, stress and anxiety manifested by the players. Practical suggestions derived from the study are proposed.
\end{abstract}

Keywords: Perfectionism, mental health, football, youth, sports categories.

* Autor de correspondencia: Aurelio Olmedilla. olmedilla@um.es

Recibido: 25 de abril de 2020

Aceptado: 14 de junio de 2020

Publicado: 14 de julio de 2020

Como citar (APA): Pérez-Hernández, P., Olmedilla-Caballero, B., Gómez-Espejo, V., \& Olmedilla, A. (2020). Relación entre perfeccionismo y salud mental en futbolistas jóvenes: diferencias entre categorías deportivas. JUMP, (2), 7-15. https://doi.org/10.17561/jump.n2.1

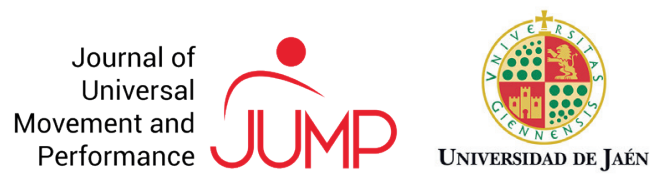




\section{Introducción}

El perfeccionismo se define como una disposición de personalidad, de carácter multidimensional, en la que se combinan unos estándares personales excesivamente altos, y una autoevaluación demasiado crítica (Frost, Marten, Lahart y Rosenblate, 1990). Tradicionalmente, las personas perfeccionistas se han visto como individuos con psicopatologías, problemas de ansiedad, depresión, desórdenes alimenticios, etc. Incluso las teorías psicodinámicas hablaban del perfeccionismo como un signo de personalidad neurótica y desordenada (Stoeber y Otto, 2006).

Tras multitud de estudios sobre el perfeccionismo, como el de Burns (1980), que estudiaba el perfeccionismo como un constructo unidimensional, o el de Hewitt y Flett (1991), que fue el primero en proponer que se trataba de un constructo multidimensional, se crea un modelo de orden superior que incluye dos dimensiones superiores del perfeccionismo (Gotwals, Stoeber, Dunn y Stoll, 2012). Por un lado, los esfuerzos perfeccionistas, aquellos aspectos del perfeccionismo asociados a un establecimiento de estándares de desempeño personal muy altos, y aquellos esfuerzos orientados a la búsqueda de esa perfección, generándose un efecto motivador para conseguir los objetivos (Ommundsen, Roberts, Lemyre y Miller, 2005). La segunda dimensión, las preocupaciones perfeccionistas, entendiéndose como aquellos aspectos relacionados con las preocupaciones por cometer errores, el temor a una evaluación social negativa, los sentimientos que surgen cuando las expectativas de logro y el desempeño de uno discrepan, y aquellas reacciones negativas que surgen ante la imperfección (Gotwals et al., 2012). A raíz de este último modelo de orden superior de se encuentra que en realidad existen dos tipos de perfeccionismo: perfeccionismo adaptativo y perfeccionismo desadaptativo (Haase y Prapavessis, 2004), relacionándose sobre todo los esfuerzos perfeccionistas con el perfeccionismo adaptativo y las preocupaciones perfeccionistas con el perfeccionismo desadaptativo (Stoeber, 2011).

Uno de los ámbitos donde más se ha estudiado el perfeccionismo es en el deporte (Hill, MallinsonHoward y Jowett, 2018). Así, se ha estudiado la relación que guarda el perfeccionismo con síntomas de depresión, estrés o ansiedad en diversos deportes. La influencia del perfeccionismo es muy compleja, y no está exenta de controversias.
En el estudio de Hill, Witcher, Gotwals y Leyland (2015), atletas internacionales afirmaron que el perfeccionismo era para ellos una gran fuente de motivación que los ayudaba a conseguir sus objetivos, aunque también les acarreaba una serie de dificultades personales e interpersonales, como preocupaciones, ansiedad, o insomnio. Existe, por tanto, un debate acerca de si el perfeccionismo es algo que se debe fomentar o evitar.

En los últimos años está aumentando considerablemente el número de síntomas de trastornos mentales en deportistas. En una investigación llevada a cabo por FIFPro, de cada 25 futbolistas profesionales, entre 3 y 9 de ellos mostraron síntomas de trastornos mentales comunes, tales como ansiedad, estrés y depresión, durante una temporada. El 95\% de esos jugadores estuvieron de acuerdo en que esos síntomas influyen negativamente en su rendimiento, un 65\% admitió que su carrera futbolística se ha visto muy influenciada por esos síntomas, y un 84\% reconoció que no existen suficientes medidas de apoyo en su carrera futbolística para poder combatir esos síntomas problemáticos (Gouttebarge et al, 2015).

Entendida la magnitud del problema con el ejemplo del estudio anterior, existen estudios, como el de Schaal et al., (2011) en los que se trata de estudiar la relación que tiene el perfeccionismo en la presencia de síntomas de trastornos mentales, como lo son la ansiedad, la depresión o la angustia/estrés. Se ha relacionado el perfeccionismo con la ansiedad en estudios como el de Stoeber, Otto, Pescheck, Becker y Stoll, (2007), o el de Koivula, Hassmen y Falby, (2002). La relación entre perfeccionismo y depresión, aunque encontrándose discrepancias en cuanto a las estadísticas de prevalencia, se encuentra en diferentes estudios (Gulliver, Griffiths, Mackinnon, Batterham y Stanimirovic, 2015; Hewitt, Flett y Endler, 1995; Nixford, Frank y Beckmann, 2016; Schaal et al., 2011; Wolanin, Hong, Marks, Panchoo y Gross, 2016). Por último, se ha relacionado el perfeccionismo con el estrés en estudios como el de Crocker, Gaudreau, Mosewich y Kljajic (2014), Flett y Hewitt, (2005), Tashman, Tenenbaum y Eklund, (2010) y el de Hall (2006), encontrándose relación principalmente con el perfeccionismo desadaptativo, aunque en el estudio de Hill, Hall, Appleton y Kozub, (2008), se encontró que los esfuerzos perfeccionistas (perfeccionismo adaptativo) también generaban distrés. 
Por otro lado, el estudio del perfeccionismo en jóvenes deportistas es escaso, aunque en los últimos años se ha incrementado el interés de los investigadores (Atienza, Appleton, Hall, Fernández y Solá, 2020; Freire et al., 2020; Mallinson-Howard, Hill y Hall, 2019). Existe alguna evidencia de que el perfeccionismo es algo que se puede "enseñar" de padres a hijos, como lo muestra el estudio de Ablard y Parker (1997). En otro estudio se encontró que aquellos jugadores con perfeccionismo desadaptativo, cuyas metas eran en su mayoría de rendimiento, a menudo tenían una mala relación con sus compañeros de equipo, no se sentían aceptados, y por tanto se interesaban menos en esforzarse para que el equipo jugara bien y consiguiera sus objetivos (Ommundsen et al., 2005). Se necesitan, sin embargo, muchos más estudios en los que se comprueben datos como la prevalencia de perfeccionismo en jóvenes deportistas, y la relación entre ese perfeccionismo y síntomas de trastornos mentales.

Teniendo en cuenta que cada año, más deportistas manifiestan tener problemas de salud mental (Frank, Nixdorf y Beckmann, 2013; Gouttebarge et al., 2015), es de gran importancia realizar estudios en esta dirección. Además, puesto que este tipo de problemas suelen empezar desde edades tempranas, parece necesario un estudio con jóvenes deportistas, en este caso jugadores de fútbol base.

El objetivo del presente estudio es estudiar la relación entre el perfeccionismo y la salud mental (depresión, estrés y ansiedad) en jóvenes futbolistas, y ver si hay diferencias en esta relación entre tres categorías deportivas (infantil, cadete y juvenil). Concretamente se pretende:

1) Determinar las diferencias en los niveles de perfeccionismo (adaptativo y desadaptativo) según la categoría deportiva: Infantil (12-13 años) Cadete (14-15 años) y Juvenil (16-18 años).

2) Determinar la relación entre el perfeccionismo (adaptativo vs. desadaptativo) y los indicadores de salud mental (Depresión, Ansiedad y Estrés) tanto en categoría Infantil, Cadete y Juvenil, como en el total de la muestra.

\section{Método}

\section{Participantes}

La muestra del estudio estuvo formada por 131 jugadores (varones) de fútbol base, de equipos pertenecientes a clubes de fútbol de la Región de
Murcia. La media de edad de los jugadores fue de 14,31 años $( \pm 1,42)$. De esos 131 futbolistas, 50 de ellos jugaban en categoría infantil (12-13 años), 44 en categoría cadete (14-15 años), y 37 en categoría juvenil (16-18 años).

\section{Instrumentos de medida}

Para evaluar las características sociodemográficas y deportivas de los jugadores se utilizó un Cuestionario ad hoc sobre variables personales y deportivas, con 14 ítems: nombre, edad, sexo, deporte, selección (si han jugado en la selección autonómica alguna vez), fecha actual, club, puesto de juego, categoría en la que compite, máxima categoría que ha competido, $n^{\circ}$ de años en la máxima categoría, $n^{\circ}$ de años haciendo deporte federado de forma continua, días de entrenamiento a la semana y horas de entrenamiento al día.

Para evaluar el perfeccionismo se utilizó la Escala Multidimensional del Perfeccionismo (MPS). Es una versión en castellano de la escala Multidimensional Perfectionism Scale de Frost et al. (1990), llevada a cabo por Carrasco, Belloch y Perpiñá (2010). Este cuestionario consta de 35 afirmaciones, en la que los sujetos deben mostrar su grado de acuerdo/ desacuerdo, siguiendo una escala tipo Likert que va de 1 (Total desacuerdo) a 5 (Completamente de acuerdo). Estas afirmaciones son aplicables a todos los contextos de la vida del sujeto, como pueden ser el deporte, los estudios, etc. El cuestionario está compuesto por 6 subescalas que se corresponden con las dimensiones del perfeccionismo. En primer lugar, hay cuatro subescalas de primer orden: Miedo a errores, Influencias paternas, Expectativas de logro, y Organización. Las otras dos subescalas son las de segundo orden: perfeccionismo adaptativo (PA) y perfeccionismo desadaptativo (PD), con cuya suma se obtiene el índice de perfeccionismo total (PT) de esa persona. La consistencia interna de este cuestionario (coeficiente a de Cronbach) es alta. Para la escala total es de 0,93, y para las cuatro subescalas son: Miedo a errores: $a=0.88$, Influencias paternas: $\mathrm{a}=0.90$, Expectativas de logro: $a=0.87$ y Organización: $a=0.89$. Las correlaciones entre los factores y la puntuación total son todas significativas $(p \leq 0.01)$. Las correlaciones de los factores entre sí también son significativas $(p \leq 0.01)$ menos la correlación entre Influencias paternas y Expectativas de logro $(r=0.12 ; p=0.05)$. (Carrasco et al., 2010). 
Para evaluar los indicadores de salud mental se utilizó la Escala de Depresión, Ansiedad y Estrés (DASS-21). Este cuestionario, de Mitchell, Burns y Dorstyn, (2008), se compone de 21 ítems, y su adaptación al castellano, que es la utilizada en este estudio, se llevó a cabo por Antúnez y Vinet (2012). Los 21 ítems se dividen en tres subescalas que miden cosas diferentes: Ansiedad, Estrés, y Depresión. La escala, de tipo Likert, va de 0 "No se aplicó a mí en absoluto" a 3 "Se aplicó mucho a mí, o la mayoría del tiempo". En este cuestionario, las afirmaciones solo son aplicables en el contexto deportivo del sujeto (en este caso fútbol), en los últimos 7 días. Las puntuaciones de las subescalas se obtienen sumando los ítems de cada una de ellas. La validez de constructo, realizada mediante un análisis factorial, compuso una estructura de tres factores que explicaban un 49.99\% de la varianza total. Su consistencia interna (coeficiente a de Cronbach) fue alta también. La consistencia interna del DASS-21, que se obtuvo mediante el coeficiente a de Cronbach fue elevada $(a=0.91)$. La consistencia interna de cada uno de los factores fueron las siguientes: Depresión: $a=0.85$, Estrés: $a=0.83$ y Ansiedad: $a=0.73$ (Antúnez y Vinet, 2012).

\section{Procedimiento}

Para la realización de este estudio, se contactó con dos de los coordinadores de fútbol base de la Federación de Fútbol de la Región de Murcia (FFRM), quienes proporcionaron los números de teléfono de los entrenadores de los distintos equipos, para poder fijar fecha, lugar y hora en los que se pasarían los cuestionarios. Una vez allí, se esperaba a que los jugadores estuvieran concentrados y atentos, y acto seguido se les explicaba el objetivo del estudio, y se les garantizaba la confidencialidad de sus datos.

Explicado esto, se daban las instrucciones de los cuestionarios en sí, haciendo hincapié en aquellos ítems en los que los sujetos pudieran tener alguna duda. El tiempo que se tardaba en completar todos los instrumentos de evaluación oscilaba entre 20 y 25 minutos. En la última página se encontraba el consentimiento informado, mediante el cual los sujetos aceptaban participar en el estudio voluntariamente. A los menores de 18 años se les entregaba otra hoja de consentimiento que sus padres debían rellenar, firmar, y hacer llegar al investigador. El estudio se realizó respetando los acuerdos de la Declaración de Helsinki en su revisión de octubre del año 2000, elaborada por la Asociación Médica Mundial (http://www.wma. net/), y fue aprobado por el Comité de Ética de la Universidad de Murcia, con código CEI-2623.

\section{Análisis estadístico}

Los datos fueron codificados y analizados con el paquete estadístico IBM SPSS Stadistic (versión 22). Se realizó un análisis descriptivo de las variables y un análisis de correlación de Pearson para determinar su relación.

\section{Resultados}

Como puede observarse en la Tabla 1, tanto los niveles de PT, como sus dos tipos (PA y PD), son muy parecidos en las tres categorías deportivas. Los niveles más altos de PT se han obtenido en la categoría Juvenil, pero con diferencias mínimas respectoalas otras dos categorías $(M=48.06 \pm 5.58)$. En cuanto a los dos tipos de perfeccionismo, se observa que los juveniles obtienen niveles un poco mayores de PA $(M=24.81 \pm 3.80)$ y $P D$ $(M=23.24 \pm 4.08)$.

En cuanto al total de la muestra, en la Tabla 2 se puede observar como el PA no se relaciona con ninguno de los indicadores de salud mental (Depresión, Ansiedad y Estrés). Por su parte, el PD muestra relación con los tres: Depresión: $F=0.33$; $p<$.05., Ansiedad: $F=0.277 ; p<.05$., Estrés: $F=0.350$; $p<.05$. Por último, se observa como el PT se relaciona con Depresión: $F=0.185, p<.01$, y con Estrés: $F=0.295 ; p<.05$, pero no muestra relación con la Ansiedad.

En categoría cadete, tal y como se puede observar en la Tabla 3, el PA no guarda relación con ningún indicador de salud mental. Por su parte, el PD se relaciona con Depresión: $F=0,346$; $p<.01$, con Ansiedad: $F=0.386 ; p<.05$, y con Estrés: $F=0.440 ; p<$.05. Por último, el PT muestra una relación con Estrés: $F=0.353 ; p<.01$.

\section{Discusión}

El objetivo principal de este estudio ha sido estudiar la relación entre el perfeccionismo y los indicadores de salud mental en jóvenes futbolistas. Concretamente los objetivos específicos han sido: a) determinar las diferencias entre las categorías de fútbol base (Infantil, Cadete y Juvenil) respecto a los niveles de perfeccionismo (adaptativo y 
Tabla 1. Análisis descriptivo de tendencia central y dispersión de la variable Perfeccionismo

\begin{tabular}{|c|c|c|c|c|c|c|c|c|c|}
\hline & & \multirow[b]{2}{*}{$\mathrm{N}$} & \multirow[b]{2}{*}{ Media } & \multirow[b]{2}{*}{$\begin{array}{l}\text { Desviación } \\
\text { típica }\end{array}$} & \multirow[b]{2}{*}{$\begin{array}{l}\text { Error } \\
\text { típico }\end{array}$} & \multicolumn{2}{|c|}{$\begin{array}{l}\text { Intervalo de confianza } \\
\text { para la media al 95\% }\end{array}$} & \multirow[b]{2}{*}{ Mínimo } & \multirow[b]{2}{*}{ Máximo } \\
\hline & & & & & & $\begin{array}{l}\text { Límite } \\
\text { inferior }\end{array}$ & $\begin{array}{l}\text { Límite } \\
\text { superior }\end{array}$ & & \\
\hline \multirow{4}{*}{ Perfeccionismo Adaptativo } & Infantil & 47 & 24,39 & 3,51 &, 512 & 23,36 & 25,42 & 17 & 33 \\
\hline & Cadete & 39 & 23,68 & 4,29 &, 688 & 22,29 & 25,07 & 16 & 32 \\
\hline & Juvenil & 35 & 24,81 & 3,80 &, 643 & 23,51 & 26,12 & 17 & 33 \\
\hline & Total & 121 & 24,29 & 3,86 &, 351 & 23,59 & 24,98 & 16 & 33 \\
\hline \multirow{4}{*}{$\begin{array}{l}\text { Perfeccionismo } \\
\text { Desadaptativo }\end{array}$} & Infantil & 47 & 23,00 & 4,62 &, 6736 & 21,64 & 24,35 & 14,5 & 35,0 \\
\hline & Cadete & 39 & 21,67 & 4,93 &, 7895 & 20,06 & 23,26 & 13,0 & 32,0 \\
\hline & Juvenil & 35 & 23,24 & 4,08 & ,6895 & 21,84 & 24,64 & 15,0 & 31,0 \\
\hline & Total & 121 & 22,64 & 4,59 &, 4171 & 21,81 & 23,46 & 13,0 & 35,0 \\
\hline \multirow{4}{*}{ Perfeccionismo Total } & Infantil & 47 & 47,39 & 6,57 & ,95806 & 45,46 & 49,32 & 36,00 & 62,50 \\
\hline & Cadete & 39 & 45,35 & 6,53 & 1,04635 & 43,22 & 47,46 & 34,50 & 59,00 \\
\hline & Juvenil & 35 & 48,06 & 5,58 & ,94286 & 46,14 & 49,97 & 36,50 & 61,00 \\
\hline & Total & 121 & 46,93 & 6,34 &, 57600 & 45,78 & 48,06 & 34,50 & 62,50 \\
\hline
\end{tabular}

Tabla 2. Relación entre Perfeccionismo e indicadores de salud mental en el total de la muestra

\begin{tabular}{|c|c|c|c|c|c|c|c|}
\hline & & PA & PD & PT & DEPRESIÓN & ANSIEDAD & ESTRÉS \\
\hline \multirow{3}{*}{$\begin{array}{l}\text { Perfeccionismo } \\
\text { Adaptativo }\end{array}$} & Correlación de Pearson & 1 & ,119 &, $695(* \star)$ &,- 088 &,- 063 &, 068 \\
\hline & Sig. (bilateral) & & ,192 &, 000 &, 335 &, 494 &, 456 \\
\hline & $\mathrm{N}$ & 121 & 121 & 121 & 121 & 121 & 121 \\
\hline \multirow{3}{*}{$\begin{array}{l}\text { Perfeccionismo } \\
\text { Desadaptativo }\end{array}$} & Correlación de Pearson & ,119 & 1 &, $797(\star \star)$ &, $330(* \star)$ &, $277(\star \star)$ &, $350(* \star)$ \\
\hline & Sig. (bilateral) & ,192 & &, 000 &, 000 &, 002 &, 000 \\
\hline & $\mathrm{N}$ & 121 & 121 & 121 & 121 & 121 & 121 \\
\hline \multirow{3}{*}{$\begin{array}{l}\text { Perfeccionismo } \\
\text { Total }\end{array}$} & Correlación de Pearson &, $695(* *)$ &, $797(* *)$ & 1 &, $185(*)$ & , 163 &, $295(* *)$ \\
\hline & Sig. (bilateral) &, 000 &, 000 & &, 042 &, 075 & ,001 \\
\hline & N & 121 & 121 & 121 & 121 & 121 & 121 \\
\hline \multirow{3}{*}{ DEPRESIÓN } & Correlación de Pearson &,- 088 &, $330(* \star)$ &, $185(\star)$ & 1 &, $474(\star \star)$ &, $486(\star *)$ \\
\hline & Sig. (bilateral) & ,335 &, 000 &, 042 & &, 000 &, 000 \\
\hline & N & 121 & 121 & 121 & 121 & 121 & 121 \\
\hline \multirow{3}{*}{ ANSIEDAD } & Correlación de Pearson &,- 063 &, $277(* \star)$ & , 163 &, $474(\star \star)$ & 1 &, $660(* \star)$ \\
\hline & Sig. (bilateral) &, 494 &, 002 &, 075 &, 000 & &, 000 \\
\hline & N & 121 & 121 & 121 & 121 & 121 & 121 \\
\hline \multirow{3}{*}{ ESTRÉS } & Correlación de Pearson &, 068 &, $350(* *)$ &, $295(* *)$ &, $486(* *)$ &, $660(* \star)$ & 1 \\
\hline & Sig. (bilateral) &, 456 &, 000 &, 001 &, 000 &, 000 & \\
\hline & $\mathrm{N}$ & 121 & 121 & 121 & 121 & 121 & 121 \\
\hline
\end{tabular}

** La correlación es significativa al nivel 0,01 (bilateral).

* La correlación es significante al nivel 0,05 (bilateral). 
Tabla 3. Relación entre Perfeccionismo e indicadores de salud mental en Infantiles

\begin{tabular}{|c|c|c|c|c|c|c|c|}
\hline & & PA & PD & PT & DEPRESIÓN & ANSIEDAD & ESTRÉS \\
\hline \multirow{3}{*}{$\begin{array}{l}\text { Perfeccionismo } \\
\text { Adaptativo }\end{array}$} & Correlación de Pearson & 1 & ,293(*) &, $740(* *)$ &,- 140 &,- 028 & 081 \\
\hline & Sig. (bilateral) & &, 046 &, 000 &, 347 &, 851 &, 590 \\
\hline & $\mathrm{N}$ & 47 & 47 & 47 & 47 & 47 & 47 \\
\hline \multirow{3}{*}{$\begin{array}{l}\text { Perfeccionismo } \\
\text { Desadaptativo }\end{array}$} & Correlación de Pearson &, $293(\star)$ & 1 &, $860(* *)$ &, $346(*)$ &, $386(\star \star)$ &, $440(* *)$ \\
\hline & Sig. (bilateral) &, 046 & &, 000 &, 017 &, 007 &, 002 \\
\hline & $\mathrm{N}$ & 47 & 47 & 47 & 47 & 47 & 47 \\
\hline \multirow{3}{*}{$\begin{array}{l}\text { Perfeccionismo } \\
\text { Total }\end{array}$} & Correlación de Pearson &, $740(* \star)$ &, $860(\star \star)$ & 1 & , 169 &, 256 &, $353(*)$ \\
\hline & Sig. (bilateral) &, 000 &, 000 & &, 258 & ,082 &, 015 \\
\hline & $\mathrm{N}$ & 47 & 47 & 47 & 47 & 47 & 47 \\
\hline \multirow{3}{*}{ DEPRESIÓN } & Correlación de Pearson &,- 140 &, $346(*)$ & , 169 & 1 &, $583(* \star)$ &, $533(* *)$ \\
\hline & Sig. (bilateral) &, 347 &, 017 &, 258 & &, 000 &, 000 \\
\hline & $\mathrm{N}$ & 47 & 47 & 47 & 47 & 47 & 47 \\
\hline \multirow{3}{*}{ ANSIEDAD } & Correlación de Pearson &,- 028 &, $386(\star \star)$ &, 256 &, $583(\star \star)$ & 1 &, $729(* *)$ \\
\hline & Sig. (bilateral) &, 851 &, 007 & ,082 &, 000 & &, 000 \\
\hline & N & 47 & 47 & 47 & 47 & 47 & 47 \\
\hline \multirow{3}{*}{ ESTRÉS } & Correlación de Pearson & ,081 &, $440(* \star)$ &, $353(*)$ &, $533(* \star)$ &, $729(\star \star)$ & 1 \\
\hline & Sig. (bilateral) &, 590 &, 002 & 015 &, 000 &, 000 & \\
\hline & $\mathrm{N}$ & 47 & 47 & 47 & 47 & 47 & 47 \\
\hline
\end{tabular}

** La correlación es significativa al nivel 0,01 (bilateral).

* La correlación es significante al nivel 0,05 (bilateral).

desadaptativo) manifestado por los jugadores; y b) establecer la relación entre los indicadores de salud mental (Depresión, Ansiedad y Estrés) y el perfeccionismo (adaptativo vs. desadaptativo) tanto para el total de la muestra, como para cada una de las tres categorías estudiadas (Infantil, Cadete y Juvenil).

Respecto al primer objetivo específico, los resultados muestran que los niveles de Perfeccionismo Total son muy parecidos en todas las categorías deportivas (y por tanto en todas las edades). Se observa lo mismo en los dos tipos de Perfeccionismo estudiados (Adaptativo y Desadaptativo). Estos hallazgos siguen la línea del estudio de Stoeber y Stoeber (2009), en el que los investigadores indicaron que ser perfeccionista, tanto de forma global, como en alguna de las dimensiones individuales de perfeccionismo, no se relacionaba con la edad. En cualquier caso, estudios como el de González-Hernández, Gómez-López, Alarcón-García y Muñoz-Villena (2019) indican la importancia de establecer de forma precisa las relaciones entre perfeccionismo (adaptativo y desadaptativo) y la práctica deportiva en adolescentes, permitiendo así el diseño de programas para que éstos sepan cómo afrontar situaciones interpretadas como estresantes de manera efectiva. Un avance en este sentido se puede considerar la validación en deportistas jóvenes españoles del Inventario Multidimensional de Perfeccionismo en Deporte (MIPS) de Atienza et al. (2020), que permite analizar su asociación con la ansiedad competitiva y la autoestima, entre otras variables.

Respecto al segundo objetivo específico, los resultados muestran que, respecto al total de la muestra, el Perfeccionismo adaptativo no se relaciona con ninguno de los indicadores de salud mental, ni con la categoría deportiva. Estos hallazgos están en la línea de lo encontrado por otros estudios; así, aquellos en los que el Perfeccionismo adaptativo no se relaciona con emociones negativas (aquellas que provocan a la larga problemas mentales) tales como enojo ante el error, o ansiedad cognitiva (GonzálezHernández y González-Reyes, 2017; Gotwals et al., 2012), ni dudas acerca de la propia capacidad, o preocupación por las expectativas de otras personas (Gould, Dieffenbach y Moffett, 2002). Es más, otros estudios aseguran que este tipo de Perfeccionismo se relaciona con variables adaptativas y beneficiosas para las personas, como tener una orientación a la tarea, o confianza en uno mismo (Stoeber, 2011), si bien el papel que, 
en este sentido, pueden jugar los entrenadores es clave para facilitar esta relación (Pineda-Espejel, López-Walle y Tomás, 2017).

El Perfeccionismo desadaptativo, por su parte, sigue un patrón totalmente opuesto. En los datos del total de la muestra, se observa como el Perfeccionismo desadaptativo se relaciona positivamente con los indicadores de salud mental (Depresión, Ansiedad, y Estrés), es decir, a mayor puntuación en Perfeccionismo desadaptativo mayores niveles de depresión, de estrés y de ansiedad. Estos hallazgos están en la línea de lo encontrado por otros estudios (GonzálezHernández y Muñoz-Villena, 2019; Muñoz-Villena, González y Olmedilla, 2016; Stoeber, 2011). Las variables desadaptativas y el Perfeccionismo desadaptativo, en general, se asocian a miedo al fracaso, orientación de meta, y por tanto a deportistas que pueden sentirse inferiores a sus rivales cuando pierden, o manifestar sentimientos de incompetencia y de ansiedad, mostrando una mayor motivación extrínseca. A su vez, las dimensiones desadaptativas del Perfeccionismo también han mostrado relación en otros estudios con otras emociones negativas, como una menor autoestima, y mayor ira (Muñoz-Villena et al., 2016). Por otro lado, respecto a la categoría deportiva los resultados muestran que, solo en la categoría infantil, aparece relación entre Perfeccionismo desadaptativo y ansiedad, es decir a mayor puntuación en perfeccionismo desadaptativo mayor nivel de ansiedad, pero no en cadetes ni en juveniles. Estos hallazgos son similares a lo encontrado por Jensen, Ivarsson, Fallby, Dankers y Elbe (2018) en su estudio. Jensen et al. (2018) estudiaron futbolistas de élite junior y profesionales, y hallaron que a mayor edad y categoría (profesionales frente a junior) menores niveles de ansiedad, de preocupaciones perfeccionistas.

Además, en cuanto a la relación entre Perfeccionismo desadaptativo y estrés, los resultados muestran que, tanto en la categoría infantil como en la cadete, existen relaciones en la línea de lo encontrado en otros estudios, que muestran que el Perfeccionismo desadaptativo es un buen predictor de altos niveles de estrés y de ansiedad (Jensen et al., 2018). Incluso algunos estudios, como el de Hill, Hall y Appleton (2010) los resultados muestran que el perfeccionismo desadaptativo es un buen predictor del burnout (agotamiento del deportista), con las emociones negativas asociadas que esto supone. Sin embargo, en categoría juvenil no aparece ninguna relación entre perfeccionismo, ni adaptativo ni desadaptativo, con los indicadores de salud mental, dato que contrasta con lo encontrado en otros estudios (Jensen et al., 2018; Madigan, Stoeber y Passfield, 2017). Así, Madigan et al. (2017), en un estudio con 141 deportistas juveniles (entre 16 y 19 años) que competían en diferentes deportes (60 en fútbol, 36 en rugby, 18 en baloncesto, 14 en atletismo y 13 en otros deportes), encontraron que el Perfeccionismo desadaptativo se relacionaba con altos niveles de estrés (sobre todo angustia por el entrenamiento, y dificultad para entrenar). Jensen et al. (2018) encontraron relación entre preocupaciones perfeccionistas y depresión en la muestra de deportistas junior.

Por último, y respecto al Perfeccionismo total, los resultados del presente estudio muestran que se relaciona con la depresión y el estrés en las categorías infantil y cadete, pero no en la juvenil. El estrés es, por tanto, el indicador que más relación presenta con el Perfeccionismo total, en la línea de otros estudios (González-Hernández y MuñozVilla, 2019).

\section{Limitaciones y propuestas de investigaciones futuras}

Quizá una de las limitaciones del estudio es el pequeño número muestral, sobre todo, de la categoría juvenil. Sería bueno poder incrementar el número de participantes en cada una de las categorías deportivas lo que proporcionaría unos resultados con mayor alcance.

Por otro lado, la pertenencia a un mismo club de los participantes puede restar perspectivas de generalización, por lo que sería recomendablehacer estudios con una muestra más representativa de la realidad deportiva futbolística.

Por último, una línea de investigación muy interesante podría ser el someter a estudio la posiblerelaciónbeneficiosaentreel perfeccionismo adaptativo y otras variables adaptativas como manifestar una orientación a la tarea, o confianza en uno mismo. O seguir profundizando en estudios donde se pone a prueba el valor mediador de aspectos como la dureza mental (Cowden, Crust, Jackman y Duckett, 2019). 


\section{Conclusiones}

1. No existen diferencias en los niveles de perfeccionismo (ni total, ni adaptativo, ni desadaptativo) en función de la categoría deportiva.

2. El Perfeccionismo desadaptativo se relaciona positivamente con los indicadores de salud mental (Depresión, Ansiedad, y Estrés), es decir, a mayor puntuación en Perfeccionismo desadaptativo mayores niveles de depresión, de estrés y de ansiedad, cuando se analiza la muestra total (de 12 a 18 años).

3. El Perfeccionismo desadaptativo se relaciona con la ansiedad en la categoría infantil, es decir a mayor puntuación en perfeccionismo desadaptativo mayor nivel de ansiedad en los jugadores infantiles.

\section{Aplicaciones prácticas}

Los resultados de este estudio indican que no existen diferencias en los niveles de perfeccionismo (ni total, ni adaptativo, ni desadaptativo) en función de la categoría deportiva, lo que es importante tanto para los psicólogos deportivos, como paraentrenadores y técnicos. Este conocimiento permite el poder establecer unos estilos de liderazgo y unos procedimientos de aprendizaje deportivo por parte de entrenadores y técnicos similares para las tres categorías. En este sentido, es importante tener en cuenta que uno de los tipos de perfeccionismo, el desadaptativo sí se relaciona con indicadores de salud mental, como la depresión, la ansiedad y el estrés, y lo hace con toda la muestra, es decir, en las tres categorías estudiadas. Tal y como se indica en algunos estudios el papel del entrenador aquí es fundamental, para intentar neutralizar las situaciones y acciones que pueden provocar conductas y pensamientos excesivamente rígidos; la "perfección no existe" podría ser una máxima del entrenador, sin que ello lo aleje de intentar un aprendizaje significativo que acerque al jugador a su mejor versión. De hecho, y particularmente en la categoría infantil, se puede observar que a mayor puntuación en perfeccionismo desadaptativo mayor nivel de ansiedad en los jugadores. El entrenador deberá posibilitar un método de enseñanza deportiva constructivo, que ayude a desarrollar los aspectos positivos del perfeccionismo (adaptativo), proponiendo entrenamientos basados en trabajos orientados a la tarea, posibilitando el desarrollo de la confianza en uno mismo.

\section{Agradecimientos}

Este estudio se ha realizado, en parte, gracias al Convenio de Colaboración entre la Federación de Fútbol de la Región de Murcia (FFRM) y la Universidad de Murcia, Proyecto FFRMUMU-040092 321B 6450214704.

\section{Referencias}

Ablard, K. E., y Parker, W. D. (1997). Parents' achievement goals and perfectionism in their academically talented children. Journal of Youth and Adolescence, 26(6), 651-667. https://doi.org/10.1023/A:1022392524554

Antúnez, Z. y Vinet, E. V. (2012). Escalas de depresión, ansiedad y Estrés (DASS-21): Validación de la Versión abreviada en Estudiantes Universitarios Chilenos. Terapia Psicológica, 30(3), 49-55. http://dx.doi.org/10.4067/S0718-48082012000300005

Atienza, F., Appleton, P., Hall, H. K., Castillo, I.y Balaguer, I. (2020). Validation of the Spanish version of multidimensional inventory of perfectionism in young footballers. Cuadernos de Psicología del Deporte, 20(1), 118-129.

https://revistas.um.es/cpd/article/view/397951/277041

Ato, M., López-García, J. J., y Benavente, A. (2013). Un sistema de clasificación de los diseños de investigación en psicología. Anales de Psicología/Annals of Psychology, 29(3), 1038-1059. http://dx.doi.org/10.6018/analesps.29.3.178511

Burns, D. D. (1980). Feeling good: The new mood therapy. New York: New American Library

Carrasco, A., Belloch, A., y Perpiñá, C. (2010). La evaluación del perfeccionismo: utilidad de la Escala Multi-dimensional de Perfeccionismo en población española. Análisis y Modificación de Conducta, 36(153), 49-65.

Cowden, R. G., Crust, L., Jackman, P. C., \& Duckett, T. R. (2019). Perfectionism and motivation in sport: The mediating role of mental toughness. South African Journal of Science, 175(1-2), 1-7. https://doi.org/10.17159/sajs.2019/5271

Crocker, P. R., Gaudreau, P., Mosewich, A. D. y Kljajic, K. (2014). Perfectionism and the stress process in intercollegiate athletes: Examining the $2 \times 2$ model of perfectionism in sport competition. International Journal of Sport Psychology, 45(4), 61-84.

Flett, G. L., y Hewitt, P. L. (2005). The perils of perfectionism in sports and exercise. Current Directions in Psychological Science, 14, 14-18.

Frank, R., Nixdorf, I., y Beckmann, J. (2013). Depression in Elite Athletes: Prevalence and Psychological Factors. Deutsche Zeitschrift Fur Sportmedizin, 64(11), 320-326. https://doi.org/10.5960/dzsm.2013.088

Freire, G. L. M., da Cruz Sousa, V., Alves, J. F. N., de Moraes, J. F. V. N., de Oliveira, D. V., \& do Nascimento Junior, J. R. A. (2020). Are the traits of perfectionism associated with pre-competitive anxiety in young athletes?. Cuadernos de Psicología del Deporte, 20(2), 37-46. https://doi.org/10.6018/cpd.406031

Frost, R. O., Marten, P., Lahart, C., y Rosenblate, R. (1990). The dimensions of perfectionism. Cognitive therapy and research, 14(5), 449-468. https://doi.org/10.1007/BF01172967

González-Hernández, J. y González Reyes, A. (2017). Perfeccionismo y "alarma adaptativa" a la ansiedad en deportes de combate. Revista de Psicología del Deporte, 26(2), 141-148. 
González-Hernández, J., \& Muñoz-Villena, A. J. (2019). Perfectionism and sporting practice. Functional stress regulation in adolescence. Anales De Psicología/Annals of Psychology, 35(1), 148-155. https://www.rpd-online.com/

González-Hernández, J., Gómez-López, M., Alarcón-García, A., \& Muñoz-Villena, A.J. (2019). Perfectionism and stress control in adolescents: Differences and relations according to the intensity of sports practice. Journal of Human Sport and Exercise, 14(1), 195-206. https://doi.org/10.14198/jhse.2019.141.16

Gotwals, J. K., Stoeber, J., Dunn, J. G., y Stoll, O. (2012). Are perfectionistic strivings in sport adaptive? A systematic review of confirmatory, contradictory, and mixed evidence. Canadian Psychology/Psychologie canadienne, 53(4), 263-279. https://doi.apa.org/doi/10.1037/a0030288

Gould, D., Dieffenbach, K., y Moffett, A. (2002). Psychological characteristics and their development in Olympic champions. Journal of Applied Sport Psychology, 14(3), 172-204. https://doi.org/10.1080/10413200290103482

Gouttebarge, V., Backx, F. J., Aoki, H., y Kerkhoffs, G. M. (2015), Symptoms of common mental disorders in professional football (soccer) across five European countries. Journal of Sports Science \& Medicine, 14(4), 811-818. https://europepmc.org/

Gulliver, A., Griffiths, K. M., Mackinnon, A., Batterham, P. J., y Stanimirovic, R. (2015). The mental health of Australian elite athletes. Journal of Science and Medicine in Sport, 18(3), 255261. https://doi.org/10.1016/j.jsams.2014.04.006

Haase, A. M., y Prapavessis, H. (2004). Assessing the factor structure and composition of the Positive and Negative Perfectionism Scale in sport. Personality and Individual Differences, 36(7), 1725-1740.

Hall, H. K. (2006). Perfectionism: A hallmark quality of world class performers, or a psychological impediment to athletic development. Essential Processes for Attaining Peak Performance, 1(7), 178-211.

Hewitt, P. L., Flett, G. L., y Endler, N. S. (1995). Perfectionism, coping, and depression symptomatology in a clinical sample. Clinical Psychology \& Psychotherapy, 2(1), 47-58

Hewitt, P. L., y Flett, G. L. (1991). Perfectionism in the self and social contexts: conceptualization, assessment, and association with psychopathology. Journal of Personality and Social Psychology, 60(3), $456-470$ https://doi.apa.org/doi/10.1037/0022-3514.60.3.456

Hill, A. P., Hall, H. K., Appleton, P. R., y Kozub, S. A. (2008) Perfectionism and burnout in junior elite soccer players: The mediating influence of unconditional self-acceptance. Psychology of Sport and Exercise, 9(5), 630 - 644

Hill, A. P., Hall, H. K., y Appleton, P. R. (2010). Perfectionism and athlete burnout in junior elite athletes: The mediating role of coping tendencies. Anxiety, Stress, \& Coping, 23(4), $415-430$. https://doi.org/10.1080/10615800903330966

Hill, A. P., Mallinson-Howard, S. H., \& Jowett, G. E. (2018). Multidimensional perfectionism in sport: A meta-analytical review. Sport, Exercise, and Performance Psychology, 7(3), 235 - 270. http://dx.doi.org/10.1037/spy0000125

Hill, A. P., Witcher, C. S., Gotwals, J. K., y Leyland, A. F. (2015). A qualitative study of perfectionism among self-identified perfectionists in sport and the performing arts. Sport, Exercise, and Performance Psychology, 4(4), $237-253$. https://psycnet.apa.org/doi/10.1037/spy0000041

Jensen, S. N., Ivarsson, A., Fallby, J., Dankers, S., y Elbe, A. M. (2018). Depression in Danish and Swedish elite football players and its relation to perfectionism and anxiety. Psychology of Sport and Exercise, 36, $147-155$ https://doi.org/10.1016/j.psychsport.2018.02.008
Koivula, N., Hassmén, P., y Fallby, J. (2002). Self-esteem and perfectionism in elite athletes: Effects on competitive anxiety and selfconfiden-ce. Personality and Individual Differences, 32(5), 865-875. https://psycnet.apa.org/doi/10.1016/S0191-8869(01)00092-7

Madigan, D. J., Stoeber, J., y Passfield, L. (2017). Perfectionism and training distress in junior athletes: a longitudinal investigation. Journal of Sports Sciences, 35(5), 470- 475 https://doi.org/10.1080/02640414.2016.1172726

Mallinson-Howard, S. H., Hill, A. P., \& Hall, H. K. (2019). The $2 \times 2$ model of perfectionism and negative experiences in youth sport. Psychology of Sport and Exercise, 45, 101581. https://doi.org/10.1016/j.psychsport.2019.101581

Mitchell, M. C., Burns, N. R., y Dorstyn, D. S. (2008). Screening for depression and anxiety in spinal cord injury with DASS-21. Spinal Cord, 46(8), 547. https://doi.org/10.1038/sj.sc.3102154

Muñoz, A. J., González, J., y Olmedilla, A. (2016). Perfeccionismo percibido y expresión de ira en deportistas varones adolescentes: Influencia sobre la autoestima. Revista Argentina de Ciencias del Comportamiento, 8(3), 30-39.

Nixdorf, I., Frank, R., y Beckmann, J. (2016). Comparison of Athletes' Proneness to Depressive Symptoms in Individual and Team Sports: Research on Psychological Mediators in Junior Elite Athletes. Frontiers in Psychology, 7, 893.

Ommundsen, Y., Roberts, G. C., Lemyre, P. N., y Miller, B. W. (2005). Peer relationships in adolescent competitive soccer: Associations to perceived motivational climate, achievement goals and perfec-tionism. Journal of Sports Sciences, 23(9), 977-989. https://doi.org/10.1080/02640410500127975

Pineda-Espejel, H. A., López-Walle, J. M. y Tomás, I. (2017). Influencia del entrenador deportivo con relación al perfeccionismo y las orientaciones de meta. Revista de Psicología del Deporte, 26(2), 141-148.

Schaal, K., Tafflet, M., Nassif, H., Thibault, V., Pichard, C., Alcotte, M., Guillet,T., El Helou, N.. Berthelot, G., Simon, S. Toussaint, J. F. (2011). Psychological balance in high level athletes: genderbased differences and sport-specific patterns. PloS one, 6(5), e19007. https://doi.org/10.1371/journal.pone.0019007

Stoeber, J. (2011). The dual nature of perfectionism in sports: Relationships with emotion, motivation, and performance. International Review of Sport and Exercise Psychology, 4(2), 128-145. https://doi.org/10.1080/1750984X.2011.604789

Stoeber, J., Otto, K., Pescheck, E., Becker, C., y Stoll, O. (2007). Perfectionism and competitive anxiety in athletes: Differentiating striving for perfection and negative reactions to imperfection. Personality and Individual Differences, 42(6) 959-969. https://doi.org/10.1016/j.paid.2006.09.006

Stoeber, J., y Otto, K. (2006). Positive conceptions of perfectionism: Approaches, evidence, challenges. Personality and Social Psychology Review, 10(4), 295-319. https://doi.org/10.1207/s15327957pspr1004_2

Stoeber, J., y Stoeber, F. S. (2009). Domains of perfectionism: Prevalence and relationships with perfectionism, gender, age, and satisfaction with life. Personality and Individual Differences, 46(4), 530-535. https://doi.org/10.1016/j.paid.2008.12.006

Tashman, L. S., Tenenbaum, G. y Eklund, R. (2010). The effect of perceived stress on the relationship between perfectionism and burnout in coaches. Anxiety, Stress, \& Coping, 23(2), 195212. https://doi.org/10.1080/10615800802629922

Wolanin, A., Hong, E., Marks, D., Panchoo, K., y Gross, M. (2016). Prevalence of clinically elevated depres-sive symptoms in college athletes and differences by gender and sport. $\mathrm{Br} J$ Sports Med 50(3), 167-171. http://dx.doi.org/10.1136/bjsports-2015-095756 